\title{
Community-driven Social Influence Analysis and Applications
}

\author{
Yang Zhang $^{1 \star}$ and Jun Pang ${ }^{1,2}$ \\ 1 Faculty of Science, Technology and Communication \\ 2 Interdisciplinary Centre for Security, Reliability and Trust \\ University of Luxembourg \\ yang.zhang@uni.lu, jun.pang@uni.lu
}

\begin{abstract}
Nowadays, people conduct a lot of activities with their online social networks. With the large amount of social data available, quantitative analysis of social influence becomes feasible. In this $\mathrm{PhD}$ project, we aim to study users' social influence at the community level, mainly because users in social networks are naturally organized in communities and communities play fundamental roles in understanding social behaviors and social phenomenons. Through experiments with a location-based social networks dataset, we start by demonstrating communities' influence on users' mobility, and then we focus on the influence of leaders in the communities. As a next step, we intend to detect users that act as structural hole spanners and analyze their social influence across different communities. Based on these studies, we plan to propose a unified approach to quantify users' social influence and investigate its applications, for example, in social interaction and behavior analysis.
\end{abstract}

\section{Introduction}

Online social networks (OSNs) have gained a lot of popularity and experienced fast growth during the past decade. Leading actors in OSNs including Facebook and Twitter have a huge amount of users. Nowadays, users share many information about themselves (such as personal profiles, holiday photos and social relationships), and conduct a lot of activities with their OSNs. With these large quantity of data regarding social behaviors, studies towards understanding human society become quantitatively achievable.

Social influence as a phenomena has been extensively studied in the literature [1]. In simple terms, it refers to the behavioral change of individuals affected by others in the society. Common forms of social influence include conformity, peer pressure, leadership, etc. Understanding social influence in OSNs is important and can result in many appealing applications, such as viral marketing, disease spread and urban planning.

Previous works have focused on computational models and algorithms on social influence in OSNs. Most of these works have considered social influence in

* PhD student at the Computer Science and Communications research unit. 
the scope of the whole social graph. On the other hand, users of a social network are naturally organized in communities. Communities play a more important role than friends on influencing a user's behavior, i.e., the influence is normally not from all his friends but certain communities. Therefore, community is the most useful resolution to study social influence [2]. In this project, we aim to understand, model and quantify social influence at the community level.

\section{Related Work and Our Approach}

There are mainly two directions on modeling users' social influences. One is based on the social graph structure. Methods range from simple ones such as the number of friends to more sophisticated ones, such as the ones based on PageRank. The other direction to compute users' influence is through mining their behaviors in the past. For example, the authors of [3] quantify a Twitter user's influence through the number of users that retweet his tweets.

Besides quantifying users' influence, influence propagation has been studied as well. In [4], the authors have modeled a social network as a weighted graph where the weight on an edge reflects the influence between users. They propose two influence propagation models and solve the influence maximization as an optimization problem. Goyal et al. [5] have proposed three methods to compute influence on edges among users through the actions they have conducted.

One common drawback of the above methods is that the social network is considered as a whole. Instead, we believe that communities' influence play a more important role. For example, where a user goes for lunch is influenced by his colleagues while his weekend plan probably depends on his family. In a broader view, communities' effectiveness have been demonstrated in other fields such as biology and physics. Therefore, we intend to study social influence in OSNs at the community level.

To approach our project objectives, we first perform a case study on locationbased social networks to demonstrate communities' influence. Sociological theories indicate that users' positions in social networks have a large impact on their social influence. We will then focus on users who are either the community kernels (leaders) or structural hole spanners among communities. Having understood these users' behavior, we aim to propose a unified framework to quantify users' social influence.

\section{Current Results}

\subsection{Community Detection}

To study social influence at the community level, the first step would be discovering communities in a social network. Community detection algorithms have been well studied during the past decade [6] and can be roughly classified into two kinds. Algorithms of the first kind detect communities based on certain metrics of the network structure such as clique and modularity. The second kind exploits 
attributes of the nodes in the social network. Depending on the dataset we are going to use, suitable schemes will be adopted to discover communities.

\subsection{Community Influence}

The first step of the project is to demonstrate that communities indeed play an important role on influencing users' behaviors. We have performed a case study on location-based social networks $[7,8]$. The dataset we use contains both users' location information and social relationships. We first propose a metric to quantify both a user's communities' and friends' influence. Through a comparison, we find that communities indeed have a stronger influence than friends on users' mobility. In addition, we show that different communities influence a user's mobility at different time or location. We further use a logistic model to predict a user's future location based on his communities' information, and the experimental results are promising.

\subsection{Community Leader Influence}

Leadership is a common social influence form. Leaders in a community are considered to have bigger influence than other members. To understand social influence, it is crucial to understand leaders' behaviors.

To find leaders in a community, we propose an algorithm that explores both the social graph structure and the behaviors users have conducted in the past. The leaders we find on a real-life social network dataset are distinguished from other members in the community in terms of the number of events they conduct and the number of friends they have. We further conduct several event prediction tasks on whether and when a community member will conduct a certain event given that leaders have already conducted the event before.

\section{Future Work}

\subsection{Detecting Structural Hole Spanners}

It has been proposed in sociology that users who act as intermediary between different communities have more control over the the social network in information diffusion. Users of this kind are termed as structural hole spanners. To discover structural hole spanners from the social network, Lou and Tang [9] have proposed two algorithms which are purely based on graph structure. However, besides social relationships, users also conduct a lot of actions in OSNs and these actions contain rich information about users' social behaviors. Therefore, we intend to consider not only the graph structure but also users' past behaviors to detect structural hole spanners. We plan to compare the accuracy of the structural hole spanners discovered by our algorithm with the algorithms proposed in [9]. To further demonstrate the effectiveness of our algorithm, we can explore structural hole spanners to study several interesting problems in social network analysis, such as community detection and event prediction. 


\subsection{Quantifying User Influence}

Having demonstrated the communities' influence and how information are propagated in communities, we aim to quantify each user's influence in the social network by considering communities instead of the whole graph. The output of the quantification would be an influence score for each user in the network.

We start by assigning each user an intra-community influence based on his importance in his community. Intuitively, users such as leaders will receive a high score. The intra-community score can be computed based on both the social graph structure and events already conducted by the user. Then the second value we consider is how important a user is among different communities, namely inter-community influence. In this case, the previously mentioned structural hole spanners will get high scores. In the end, the social influence of a social network user is computed through his intra-community and inter-community scores. To show the effectiveness of our community-driven influence quantification method, experiments on various social network datasets will be conducted. The baseline models for comparison will include classical solutions such as PageRank.

\subsection{Applications}

Understanding social influence of users in OSNs is critical and will benefit many web applications (e.g., viral marketing) and real-life applications (e.g., urban planning). OSN operators who can exploit social influence to improve their quality of service. For example, our new social influence analysis can lead to the design of better event and venue recommender systems for OSNs to attract users. In a broader context, a suitable social influence model will also help to attack important problems, such as monitoring and controling disease spread.

\section{References}

1. Tang, J., Chang, Y., Liu, H.: Mining social media with social theories: a survey. SIGKDD Explorations Newsletter 15(2) (2014) 20-29

2. Yang, J., McAuley, J.J., Leskovec, J.: Detecting cohesive and 2-mode communities indirected and undirected networks. In: Proc. WSDM, ACM (2014) 323-332

3. Bakshy, E., Hofman, J.M., Mason, W.A., Watts, D.J.: Everyone's an influencer: quantifying influence on twitter. In: Proc. WSDM, ACM (2011) 65-74

4. Kempe, D., Kleinberg, J., Tardos, E.: Maximizing the spread of influence through a social network. In: Proc. KDD, ACM (2003) 137-146

5. Goyal, A., Bonchi, F., Lakshmanan, L.V.: Learning influence probabilities in social networks. In: Proc. WSDM, ACM (2010) 241-250

6. Lancichinetti, A., Fortunato, S.: Community detection algorithms: a comparative analysis. CoRR abs/0908.1062 (2010)

7. PANG, J., AND ZHANG, Y. Location prediction: communities speak louder than friends. CoRR abs/1408.1228 (2014)

8. PANG, J., AND ZHANG, Y. Exploring communities for effective location prediction. In: Proc. WWW (Companion Volume), ACM (2015)

9. Lou, T., Tang, J.: Mining structural hole spanners through information diffusion in social networks. In: Proc. WWW, ACM (2013) 825-836 